Heroinen IN DER GesCHLECHTERORDNUNG 
Ergebnisse der Frauenforschung

Band 42

Begründet und im Auftrag des Präsidenten der Freien Universität Berlin herausgegeben von

Prof. Anke Bennholdt-Thomsen, Germanistik

Elisabeth Böhmer, Soziologie

Prof. Marlis Dürkop, Sozialpädagogik

Prof. Ingeborg Falck, Medizin

Prof. Marion Klewitz, Geschichtsdidaktik

Prof. Jutta Limbach, Jura

Prof. Hans Oswald, Pädagogik

Prof. Renate Rott, Soziologie

Dr. Hanna Beate Schöpp-Schilling, Amerikanistik/Anglistik, Germanistik

Prof. Margarete Zimmermann, Romanistik

Koordination: Dr. Anita Runge 
Cornelia Plume

\section{HEROINEN IN DER}

\section{GESCHLECHTERORDNUNG}

Weiblichkeitsprojektionen bei Daniel Casper von Lohenstein und die Querelle des Femmes

Verlag J. B. Metzler

Stuttgart - Weimar 
Die Deutsche Bibliothek - CIP-Einheitsaufnahme

\section{Plume, Cornelia:}

Heroinen in der Geschlechterordnung: Weiblichkeitsprojektionen bei Daniel Casper von Lohenstein und die Querelle des Femmes / Cornelia Plume. - Stuttgart ; Weimar : Metzler, 1996

(Ergebnisse der Frauenforschung; Bd. 42)

ISBN 978-3-476-01462-7

ISBN 978-3-476-03669-8 (eBook)

DOI 10.1007/978-3-476-03669-8

NE: GT

Dieses Werk einschließlich aller seiner Teile ist urheberrechtlich geschützt. Jede Verwertung außerhalb der engen Grenzen des Urheberrechtsgesetzes ist ohne Zustimmung des Verlages unzulässig und strafbar. Das gilt insbesondere für Vervielfältigungen, Übersetzungen, Mikroverfilmungen und die Einspeicherung und Verarbeitung in elektronischen Systemen.

(C) 1996 Springer-Verlag GmbH Deutschland

Ursprünglich erschienen bei J. B. Metzlersche Verlagsbuchhandlung

und Carl Ernst Poeschel Verlag GmbH in Stuttgart 1996 


\section{INHALT}

Dank....................... 8

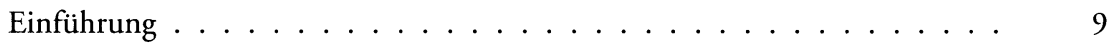

A. Die Geschlechterdiskussion innerhalb der Querelle des Femmes

I. Zugänge und Verständnisvoraussetzungen . . . . . . . . . . 15

1. Die Querelle des Femmes . . . . . . . . . . . . . . . . . . . . . 15

2. Die Querelle-Forschung . . . . . . . . . . . . . 16

3. Christine de Pizan und die Entwicklung einer weiblichen Anthropologie ..................... 20

II. Die Wurzeln neuzeitlicher misogyner Anthropologien . . . . . . . . . 21

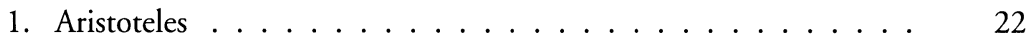

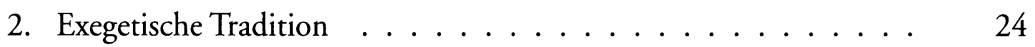

3. Säftelehre und Physiologie . . . . . . . . . . . . . . . 29

4. Weitere antike Quellen misogyner Argumentationen . . . . . 30

III. Systematische Aspekte zur Darstellung der Querelle . . . . . . . . . . 31

1. Themen und Argumentationen . . . . . . . . . . . 35

1.1 Hauptaspekt Fähigkeiten: Die Abwesenheit, männlicher' Tugenden beim weiblichen Geschlecht . . . . . . . . . . 37

1.1.1 Regierungs- und Amtsführungsfähigkeit . . . . . . . . . 37

1.1.2 Rationalität und Bildungsfähigkeit . . . . . . . . . . . 44

1.1.3 Ursachenanalyse für Misogynie und die bestehenden Verhältnisse . . . . . . . . . . . . . . . 47

1.2 Hauptaspekt Tugenden: Herausarbeitung eines weiblichen Tugendmodells . . . . . . . . . . . . . . . . . . 49

1.3 Umkehrung des Lastervorwurfs . . . . . . . . . . . . 56

B. Weiblichkeitsprojektionen im Roman.

Das Lohensteinsche Ideal vor dem Hintergrund der Querelle des Femmes

I. Interpretationszugänge . . . . . . . . . . . 57

II. Gattungskonventionen, Heroinengestaltung und die Querelle des Femmes 63

III. Strukturen des Figurenensembles . . . . . . . . . . . . . . 70

IV. Analysemodell zur Bewertung geschlechtsspezifischer Zuschreibungen . $\quad 72$

1. ,Männliche` Fähigkeiten und Tugenden beim weiblichen Geschlecht 72

1.1 Heroische Tugend. . . . . . . . . . . . . 73 
1.2 Regierungsfähigkeit . . . . . . . . . . . . . . . . . 97

1.3 Klugheit und Wissen . . . . . . . . . . . . . . . . . 110

1.4 Vaterlandsliebe . . . . . . . . . . . . . . . . 121

2. ,Weibliche Tugenden. . . . . . . . . . . . . . . . . . 126

2.1 Keuschheit . . . . . . . . . . . . . . . . . . 127

2.2 Liebe . . . . . . . . . . . . . . . 136

2.3 Treue . . . . . . . . . . . . . . . . . . 144

2.4 Gehorsam und Unterordnung . . . . . . . . . . . . . . . 149

2.5 Frömmigkeit, Gottvertrauen, Mitleid . . . . . . . . . . 163

2.6 Freundschaft . . . . . . . . . . . . . . . 167

3. Laster und Fehlverhalten weiblicher Figuren . . . . . . . . . . 173

4. Die Tugend der Geschlechter im Vergleich . . . . . . . . . 183

V. Vergleich und Ergebnisse . . . . . . . . . . . . . . . . . 186

1. Weiblichkeitsprojektionen in Herkules und Valiska von Bucholtz, Aramena von Anton Ulrich von Braunschweig-Lüneburg und $A r-$ minius und Thußnelda von Lohenstein . . . . . . . . . . . . . 186

2. Die Weiblichkeitsprojektionen im Arminius als Fürstinnenspiegel . 205

C. Weiblichkeitsprojektionen in Lohensteins Trauerspielen

I. Stand der Forschung und Fragestellung. . . . . . . . . . . . . 210

II. Funktionalisierung misogyner Ansichten $\ldots \ldots \ldots \ldots \ldots . \ldots 211$

III. Konzeptionen von Hauptfiguren … . . . . . . . . . . 221

1. Cleopatra und Augustus . . . . . . . . . . . . . . . . . 224

2. Sophonisbe . . . . . . . . . . . . . . . . . . . 227

3. Agrippina . . . . . . . . . . . . . . . 231

IV. Darstellung der weiblichen Figuren vor dem Hintergrund der QuerelleDiskussionen . . . . . . . . . . . . . . . . . . 235

Ausblick und Schluß . . . . . . . . . . . . . . . . . . . . 243

\section{Anhang}

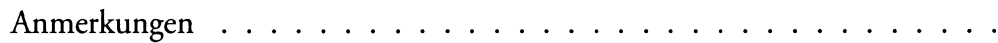

Anmerkungen zu Quellenverweisen, Siglen, Übersetzungen und Bildnachweisen

Anhang 1: Zusatzinformationen zu den in der Arbeit berücksichtigten QuerelleSchriften. 
Anhang 2: Schematische Darstellung der wichtigsten Figuren im Arminius . . 303

Anhang 3: Gegenüberstellung von Textpassagen im Arminius und in der Gallerie des femmes fortes von LeMoyne . . . . . . . . . . 304

Literaturverzeichnis . . . . . . . . . . . . . . . . . . . . 312

Quellenverzeichnis .................... 312

Sekundärliteratur . . . . . . . . . . . . . . . . . 317

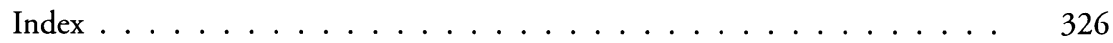




\section{DANK}

Das vorliegende Buch ist die überarbeitete Fassung einer Dissertation, die im April 1995 vom Fachbereich Germanistik der Freien Universität Berlin angenommen wurde. Viele Personen und Institutionen haben die Entstehung dieser Arbeit gefördert und begleitet. Ihnen möchte ich auf diesem Wege noch einmal herzlich danken.

Mein erster Dank gilt meinem Doktorvater, Herrn Professor Gerhard Spellerberg. Er trieb durch viele Fragen und in vielen Diskussionen dieses Vorhaben voran, förderte mich mit seiner Kritik, forderte bis ins Detail theoretisch präzise Arbeit und sprachlich genauen Ausdruck. Seinem Andenken ist dieses Buch gewidmet.

Die Anregung des Dissertationsprojekts verdanke ich Herrn Professor Horst Langer aus Greifswald, der auch die erste Arbeitsphase begleitete. Als fachkundige Ratgeber standen mir ferner Herr Professor Hans-Gert Roloff, Herr Professor Klaus Garber und Frau Professorin Barbara Becker-Cantarino zur Seite. Ihr möchte ich besonders für die Anregungen auf dem Gebiet der Frauenforschung danken.

Die Arbeit entstand überwiegend im Haus Potsdamer Straße der Staatsbibliothek Berlin und der Herzog-August-Bibliothek Wolfenbüttel. Für die jahrelange Bereitstellung, Unterstützung bei den Recherchen, besonders nach schwer aufzufindender Quellenliteratur bin ich den Mitarbeitern beider Häuser verpflichtet.

Für die Unterstützung bei der Korrektur und Fertigstellung der Dissertation danke ich Silke Ahrens, Edeltraud und Günter Heidecker, Ulrike Hansen und Robert Rutkowski, für Rat und Hilfe bei Übersetzungen Maria Rosa Antognazza, Dolly Conto Knoll, Dorothea Heidecker, Rüdiger Hillner, Hans Walter Jaenchen, Dina Kang-Euen, Nicole Kellerhals und Marion Kuschnerus.

Mein größter Dank gilt meinem Mann, Gerhard Plume, der sich viele Male in die Diskussion von Fachfragen verwickeln ließ und dessen Antworten mir häufig neue Impulse gaben. Ihm danke ich besonders für die härteste und fröhlichste Kritik, die mein Vorhaben begleitet hat.

Für die Drucklegung der Arbeit bin ich den Herausgeberinnen der Reihe „Ergebnisse der Frauenforschung “verbunden und für die Unterstützung der Endredaktion danke ich Anita Runge. 